\title{
Thixotropic behavior of paste
}

\author{
Aixiang Wu University of Science and Technology Beijing, China \\ Haiyong Cheng University of Science and Technology Beijing, China \\ Ying Yang University of Science and Technology Beijing, China
}

\begin{abstract}
Structure fluid can be broken under shear and rebuilt at the static state because of its thixotropy. The thixotropic behavior of paste slurry in transportation causes a continuous decrease of yield stress and plastic viscosity with time. There is no generally accepted method to measure the thixotropy of paste materials. In this paper, the curve of rheological properties was divided into three parts based on the rheological behavior, and a number of problems were identified using the thixotropy loops measurement to characterize the thixotropy of cement paste. Stress relaxation was considered and the variation of shear stress with time under a constant shear rate was also measured, establishing a yield stress prediction model and plastic viscosity prediction model after considering aspects of paste materials. The total thixotropic time was found to have no relevance to shear rate. Due to paste thixotropy, shear flow area increased and plunger flow area decreased over time and eventually stablized. According to the difficulty level, a standard which divides transportation into different stages was first proposed.
\end{abstract}

\section{Introduction}

Fluid thixotropy refers to the phenomenon where shear stress decreases with time under given shear rate and temperature conditions (Barnes, 1997). Using electron microscopy at high magnification, floc structures are found in fine particle slurry (Dullaert and Mewis, 2006). Due to their weak connection, structures under external force are prone to breaking (Liu et al., 2014). However, without the external force, they are also easy to rebuilt (Roussel, 2006). In a rheological test of unclassified-tailing paste filling material in Jinchuan, Wang found a significant thixotropic phenomenon (Liu et al., 2012). In the study of the rheological properties of high concentration red mud, Nguyen found that the viscosity of the slurry decreased with the shear time and finally stabilized (Usui, 1995). In the study of fine particles of slurry, Han Wenliang found a significant stress relaxation phenomenon (Wu et al., 2013).

\section{Analysis method of thixotropic properties}

The thixotropic properties of paste are affected by shear rate and time (Roussel et al., 2012), both of which are key to accurately evaluating the thixotropic properties of paste (Møller et al., 2006). With no standard method for thixotropic determination (Abebe and Lohaus, 2017), some researchers used thixotropy loop to analyze the slurry thixotropy (e.g. Mujumdar et al., 2002), as shown in Figure 1. The area between the ascending and descending curve of the thixotropy loop represents the scale of thixotropy. 


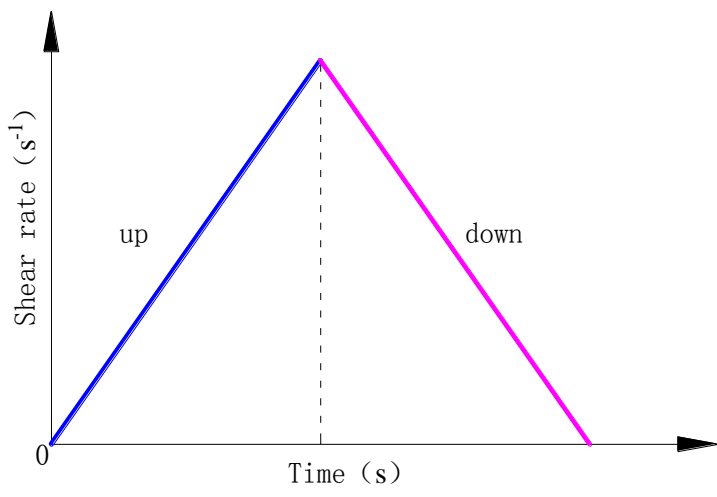

(a)

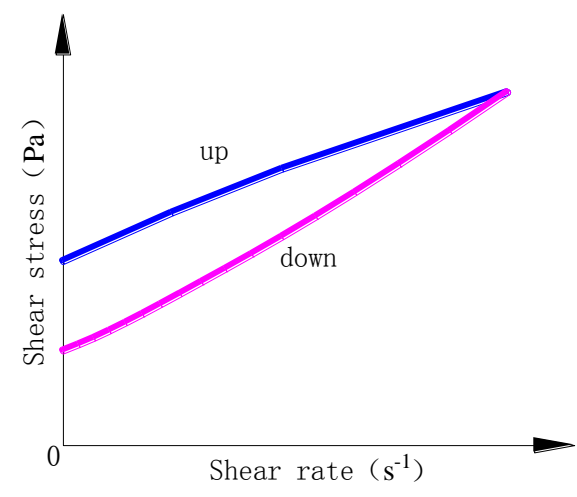

(b)

Figure 1 Diagram of thixotropy loop experiments; (a) setting program of thixotropy loop; (b) thixotropy loop

It is also found that the influence of time on thixotropy cannot be reflected by the use of thixotropy loop (Cruz and Peng, 2016). Meanwhile, the peak shear rate is also found to greatly influence the shape of thixotropy loop, especially by the downstream curve, as shown in Figure 2. If the downstream curve was used to regress the rheological parameters, the plastic viscosity after thixotropy loop experiments was often greater than the plastic viscosity before thixotropy loop experiments, opposite to the fact. The thixotropy loop could only qualitatively indicate whether the slurry has thixotropic properties, but not quantitatively describe the thixotropy scale and cannot be used to calculate the thixotropic parameters by thixotropy loop.

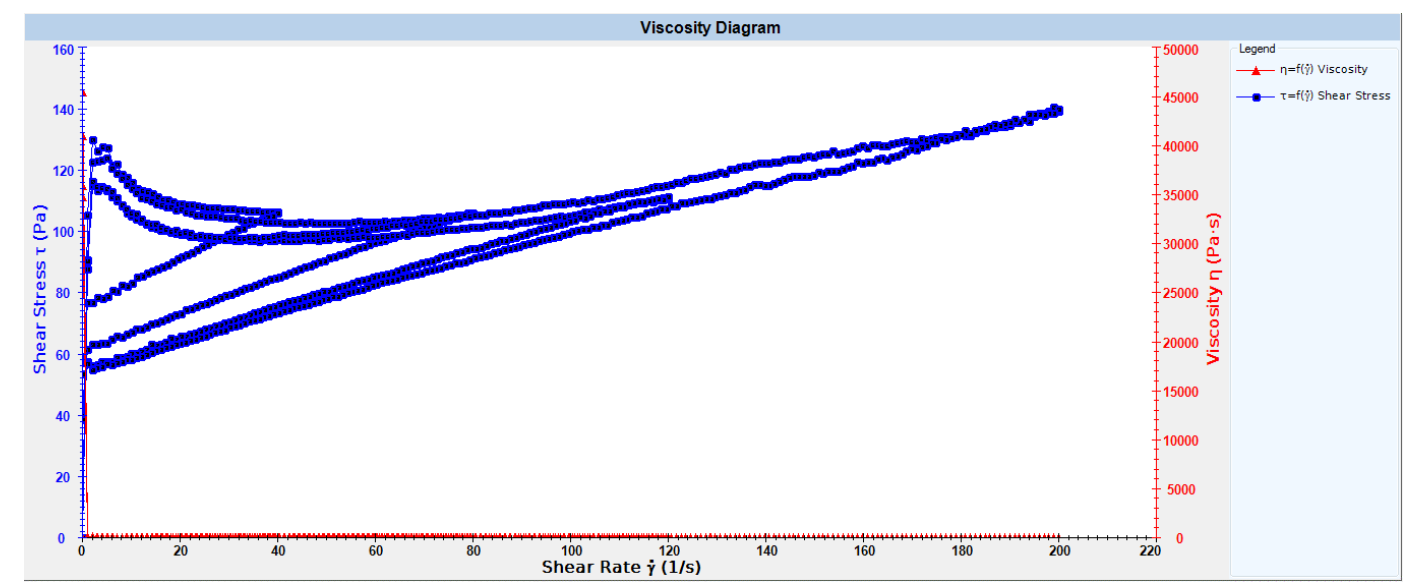

Figure 2 The results of thixotropy loop experiments

Based on a large number of exploratory experiments, it is found that the stress relaxation curve showed a certain regularity when the shear stress was fixed, and appropriate mathematical model could be used to obtain yield stress and plastic viscosity parameters before and after thixotropy loop experiments. The stress relaxation curves in Figure 3(a) show the variation of shear stress with time as well as the shear stress and stress relaxation time when relaxation was balanced. From Figure 3(b), after respectively fitting the shear stress at different shear rates before and after stress relaxation, the changing characteristics of shear stress with shear rate and yield stress and plastic viscosity after thixotropy were obtained.

This method shows both the effect of time and shear rate on thixotropy, while only requiring two or more stress relaxation tests to obtain the yield stress and plastic viscosity before and after thixotropy. Subsequent experimental design of paste thixotropy were carried out using this method. 


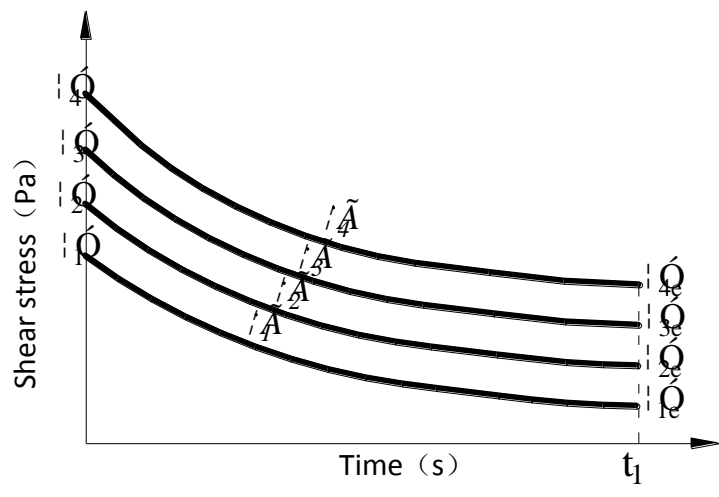

(a)

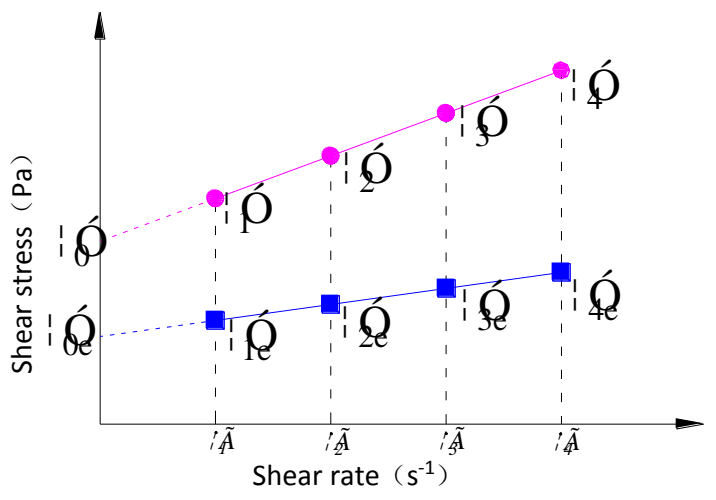

(b)

Figure 3 Calculated results of characteristic parameters of paste thixotropy using stress relaxation curve;

(a) characteristic curve of stress relaxation; (b) regression result of yield stress

\section{$3 \quad$ Thixotropic properties experiments}

\subsection{Materials and methods}

The materials used mainly included unclassified tailings and cement, which were collected from the No.2 Mine area of Jinchuan Group in western China. Prior to the experiments, materials were dehydrated in an oven at $105^{\circ} \mathrm{C}$ for 12 hours. The materials were mixed with tap water to make slurry paste with certain concentrations for different tests. The basic physical properties of the materials are shown in Table 1 . The content of -200 mesh particles of unclassified tailings was $94.7 \%$, and the $-20 \mu \mathrm{m}$ particles accounted for $21.7 \%$, meaning the overall particle size was well graded.

Table 1 Physical properties of the materials

\begin{tabular}{cccc}
\hline Type of material & Density $\left(\mathbf{t} / \mathbf{m}^{\mathbf{3}}\right)$ & Packing density $\left(\mathbf{t} / \mathbf{m}^{\mathbf{3}}\right)$ & Porosity (\%) \\
\hline Tailings & 2.85 & 1.55 & 45.61 \\
Cement & 3.1 & 1.1 & 64.52 \\
\hline
\end{tabular}

\subsection{Methods}

The samples were divided into 16 groups according to the different combinations of paste concentrations (68, 69, 70 and $71 \%)$ and cement-sand ratios (1:2, 1:4, 1:12 and 1:20). In this experiment, the shear rate was controlled as 30, 60, 90 and $120 \mathrm{~s}^{-1}$. In the preliminary exploration experiment, the test time of each group was determined on the basis of corresponding equilibrium time (Qian and Kawashima, 2016). The initial and stable data of shear stress and corresponding stress relaxation time were recorded upon the completion of the experiment (Assaad et al., 2016). 


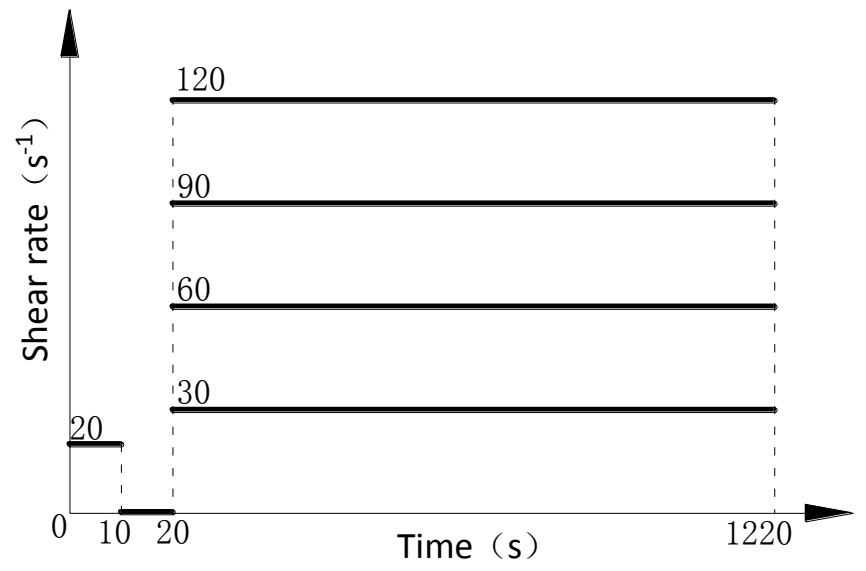

Figure 4 Setting program for measuring paste rheology during stress relaxation

\subsection{Results}

Figure 5 shows the results of the experiment when the concentration of paste was $68 \%$ and the cement sand ratio was 1:2. The initial maximum value and the stable value after thixotropy experiment of shear stress could be determined according to the stress relaxation curve in different shearing rates. The initial maximum value and the stable value after thixotropy experiment of shear stress both featured linear growth. The Bingham model was used to obtain pre-thixotropic and post-thixotropic yield stress and plastic viscosity parameter.

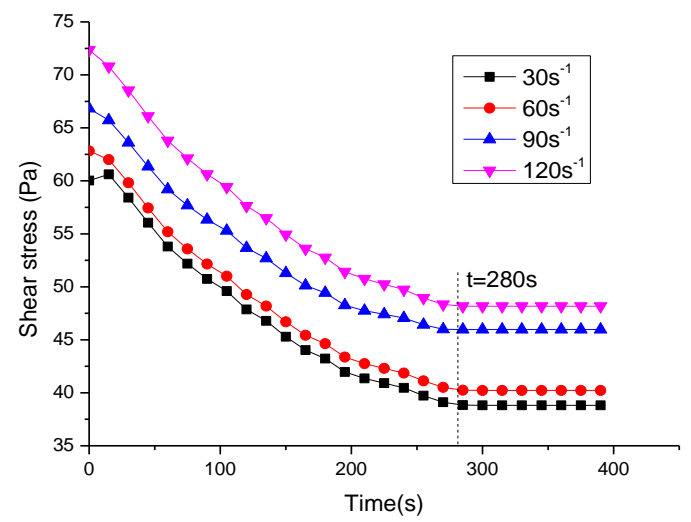

(a)

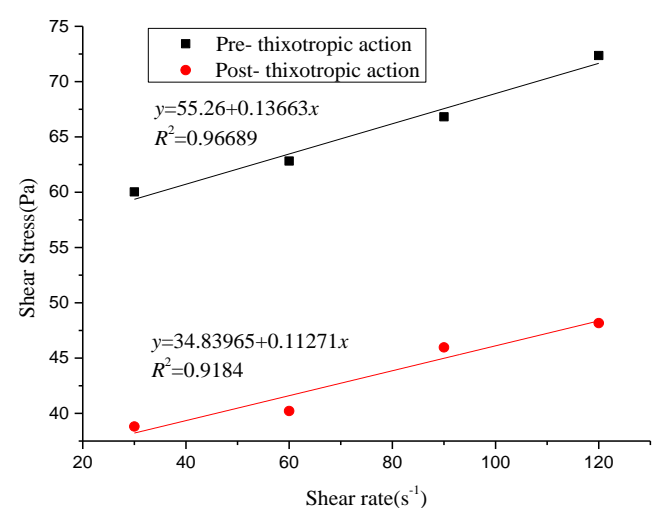

(b)

Figure 5 Stress relaxation curve and regression curve of rheological parameters in the first group (concentration was $68 \%$ and cement sand ratio was 1:2); (a) stress relaxation curve; (b) regression curve of rheological parameters (Pre-thixotropy and Post-thixotropy)

Stress relaxation time could be determined by the relationship between yield stress and time at a constant shear rate and the rheological parameters could be analyzed by the Bingham equation (Figure 5 ). The results are shown in Table 2. 
Table 2 The results of stress relaxation of different groups

\begin{tabular}{cccccccc}
\hline No. & $\begin{array}{c}\text { Concentration } \\
\text { (\%) }\end{array}$ & $\begin{array}{c}\text { Cement sand } \\
\text { ratio }\end{array}$ & $\begin{array}{c}\text { Time } \\
(\mathbf{s})\end{array}$ & $\begin{array}{c}\text { Pre-shear } \\
\text { stress } \\
(\mathbf{P a})\end{array}$ & $\begin{array}{c}\text { Post-shear } \\
\text { stress } \\
(\mathbf{P a})\end{array}$ & $\begin{array}{c}\text { Pre-plastic } \\
\text { viscosity } \\
\text { (Pa/s) }\end{array}$ & $\begin{array}{c}\text { Post-plastic } \\
\text { viscosity } \\
(\mathbf{P a} / \mathbf{s})\end{array}$ \\
\hline 1 & 68 & $1: 2$ & 280 & 55.26 & 34.84 & 0.137 & 0.113 \\
2 & 68 & $1: 4$ & 325 & 63.14 & 39.12 & 0.140 & 0.127 \\
3 & 68 & $1: 12$ & 345 & 71.93 & 42.99 & 0.161 & 0.140 \\
4 & 68 & $1: 20$ & 360 & 76.46 & 46.55 & 0.169 & 0.141 \\
5 & 69 & $1: 2$ & 470 & 69.77 & 43.09 & 0.181 & 0.161 \\
6 & 69 & $1: 4$ & 505 & 72.78 & 44.45 & 0.193 & 0.181 \\
7 & 69 & $1: 12$ & 530 & 89.12 & 56.54 & 0.201 & 0.191 \\
8 & 69 & $1: 20$ & 545 & 105.20 & 64.00 & 0.210 & 0.202 \\
9 & 70 & $1: 2$ & 880 & 96.10 & 62.33 & 0.218 & 0.209 \\
10 & 70 & $1: 4$ & 890 & 111.98 & 71.39 & 0.228 & 0.214 \\
11 & 70 & $1: 12$ & 895 & 152.94 & 99.58 & 0.243 & 0.229 \\
12 & 70 & $1: 20$ & 925 & 180.18 & 117.18 & 0.253 & 0.240 \\
13 & 71 & $1: 2$ & 1140 & 138.57 & 81.58 & 0.247 & 0.226 \\
14 & 71 & $1: 4$ & 1180 & 178.97 & 110.11 & 0.259 & 0.238 \\
15 & 71 & $1: 12$ & 1195 & 201.28 & 122.54 & 0.267 & 0.249 \\
16 & 71 & $1: 20$ & 1215 & 231.11 & 151.03 & 0.305 & 0.270 \\
\hline
\end{tabular}

\section{Discussion}

\subsection{Time variation of paste thixotropy}

The results showed a similar change law of shear stress at different shear rates. The shear stress before and after stress relaxation at high shear rate was both higher than that at the low shear rate. The thixotropic behavior of slurry was affected by the shear rate, with the amount of thixotropy increasing with the increase of shear rate. Stress relaxation curves at different shear rates showed no evident differences of relaxation time at different shear rates. In other words, while the stress relaxation rate changed at different shear rates, the stress relaxation time was similar because the difference between the initial stress state and the relaxation stress state compensated the differences of relaxation rates. Although influencing the thixotropy of paste slurry, shear rate had no obvious relation to thixotropic time, yield stress and plastic viscosity.

\subsection{Variation of yield stress}

According to stress relaxation curves of 30,60, 90 and $120 \mathrm{~s}^{-1}$ (Figure 5(a)), yield stress at each moment could be analyzed by regression. Regression analysis was carried out in every $15 \mathrm{~s}$ to optimize the analytical data. When the concentration of paste was $68 \%$ and the cement sand ratio was $1: 2$, regression analysis was performed for 26 times, to obtain the curve of yield stress during $400 \mathrm{~s}$ (Figure 6(a)). The yield stress had reached a stable state at around $280 \mathrm{~s}$. Taking the stability of the model into account, all data needed to be segmented and divided into two stages: before and after the stable state stages. The change of yield stress with time was in accordance with the negative exponential function growth property on the basis of processed data within $280 \mathrm{~s}$ (Figure 6(b)). 


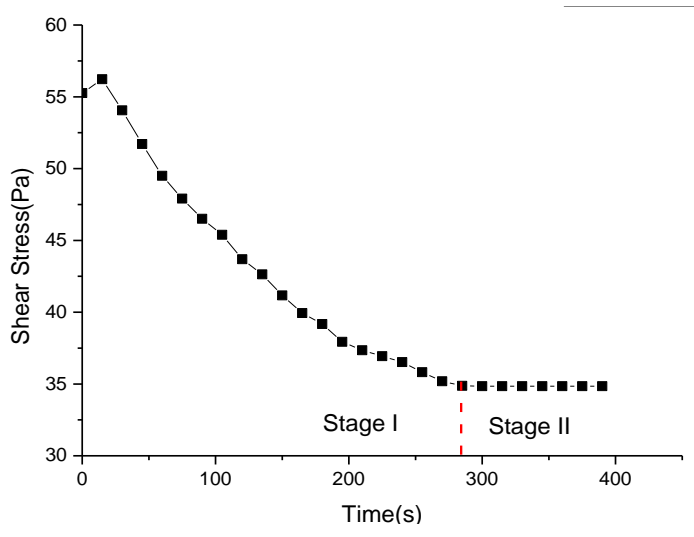

(a)

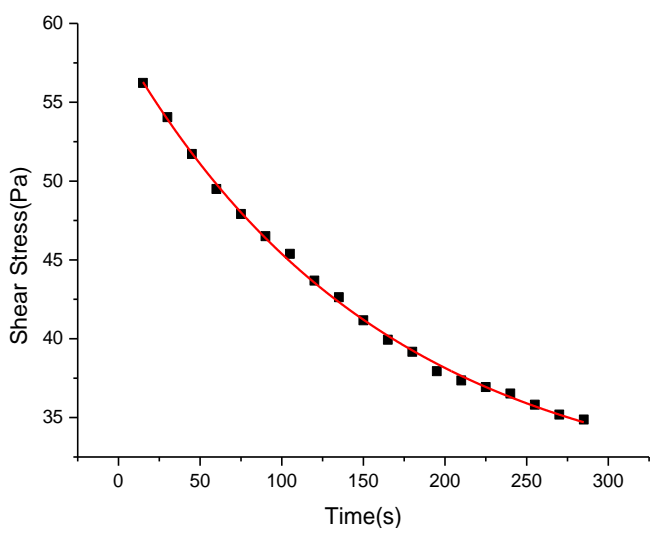

(b)

Figure 6 Change curve of yield stress with time (with the concentration of $68 \%$ and cement sand ratio of 1:2);

(a) change curve of yield stress with time; (b) fitting curve at the first stage

The variation of yield stress under thixotropic conditions was characterized by the equation below:

$$
\tau_{0}(t)=\tau_{00} \cdot \exp (-k t)
$$

Where:

$$
\begin{array}{ll}
\tau_{0} & =\text { Change value of yield stress with time, } \mathrm{Pa} ; \\
\tau_{00} & =\text { Yield stress before thixotropy, } \mathrm{Pa} \\
\mathrm{k} & =\mathrm{A} \text { parameter of thixotropic time; } \\
\mathrm{t} & =\text { Thixotropic equilibrium time, } \mathrm{s} ;
\end{array}
$$

Thixotropic time varied in different groups and the parameter of thixotropic time $(k)$ had a certain relation with the material characteristics which the author will analyze in detail in other papers.

\subsection{Variation of plastic viscosity}

The curve in Figure 7(a) shows the change of plastic viscosity (concentration was $68 \mathrm{wt} \%$ and cement-sand ratio was 1:2) with time could be divided into two stages: before and after the stable state stages. As is obviously shown in Figure 7(b), before the data were stable (280 s), plastic viscosity changed little. In addition, plastic viscosity descended by $23.59 \%$ (equal to $0.9 \%$ o per second) from the initial to the stable state.

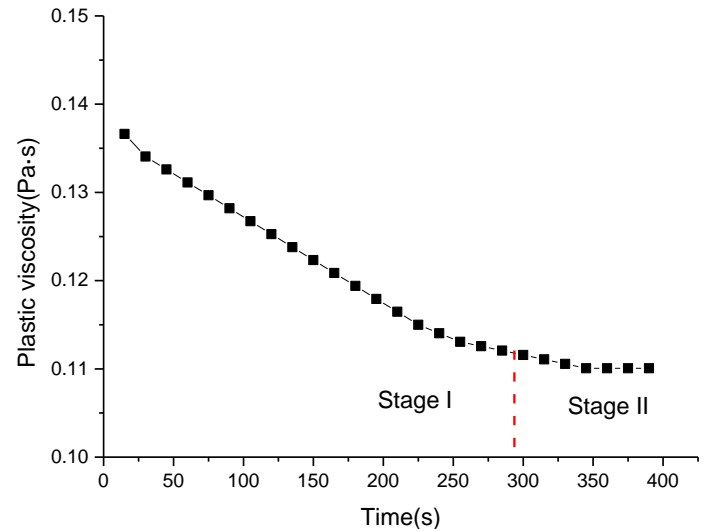

(a)

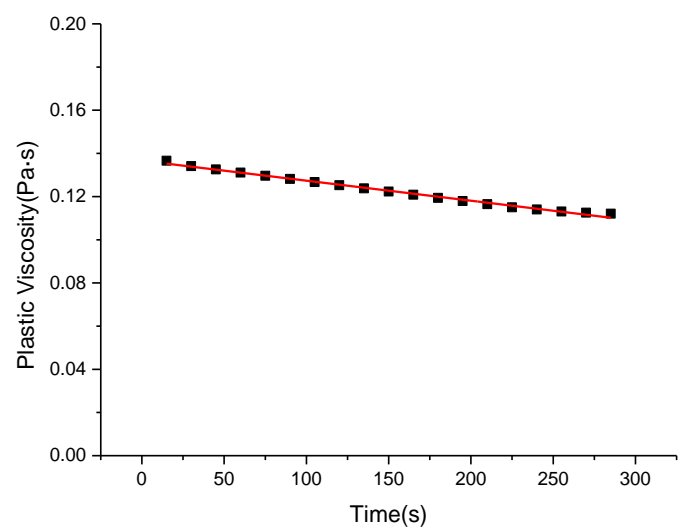

(b)

Figure 7 Change curve of plastic viscosity with time; (a) change curve of plastic viscosity with time; (b) fitting curve at the first stage

A linear function was established to express the change of plastic viscosity under the thixotropic condition.

$$
\eta_{0}(t)=\eta_{00}-m t
$$


Where:

$\eta_{0} \quad=$ Change value of plastic viscosity with time, Pa.s;

$\eta_{00}=$ Plastic viscosity before thixotropy, Pa.s;

$m$ = A parameter of thixotropic time;

$t \quad=$ Thixotropic equilibrium time, $\mathrm{s}$;

The parameter of thixotropic time $(m)$ had a certain relation with the material characteristics which the author will analyze in detail in other papers.

\subsection{Thixotropic properties of paste in transport}

As a plug flow in pipeline transportation, paste includes two types of in terms of configuration: shear flow region and slug flow region. With regard to paste thixotropy, the distribution of flow velocity is shown in Figure 8. With the passing of transport time, thixotropic properties weakened. Simultaneously, the radial range of shear flow region gradually expanded with the gradual shrink of slug flow region. The slurry and the slug flow region stabilized at time $t_{1}$, eventually leading to the stabilization of the boundary between shear flow region and slug flow region.

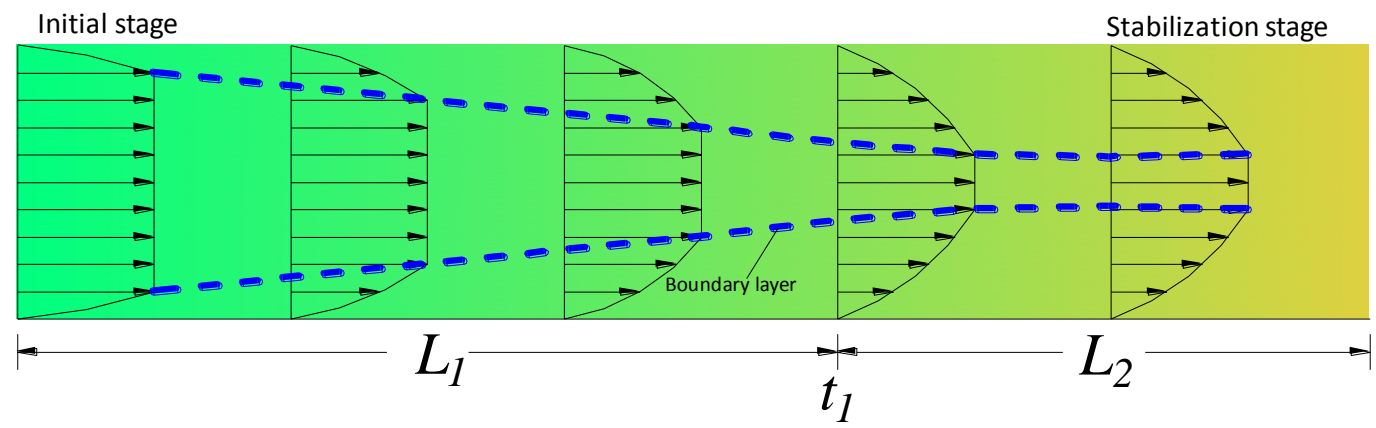

Figure 8 Distribution properties of flow velocity under thixotropic condition

\section{$5 \quad$ Conclusions}

The existence of thixotropy results in the change of the rheological properties of paste with shear rate and time. The following conclusions can be obtained:

- The thixotropy properties of paste slurry cannot be quantitatively characterized by conventional thixotropic loop which was obtained indirectly from the stress relaxation curve of slurry in consideration of time effect and shear rate.

- The total thixotropic time of paste had great relevance to the properties of paste materials, but it changed little with shear rate.

- The yield stress changed in formation of negative exponent with time and finally stabilized. In addition, the plastic viscosity had linear relationship with thixotropic time and eventually stabilized as well. When paste flowed in the pipe, the shear flow region gradually expanded and the slug flow region shrank until the thixotropic effect disappeared and the boundary layer stabilized.

\section{Acknowledgements}

This work was financially supported by the National Natural Science Foundations of China (51574013, 51374034, 51674012) 


\section{References}

Abebe, Y. A., Lohaus, L. 2017, 'Rheological characterization of the structural breakdown process to analyze the stability of flowable mortars under vibration', Construction and Building Materials, vol. 131, pp. 517-525.

Assaad, J.J., Harb, J., Maalouf, Y. 2016, 'Effect of vane configuration on yield stress measurements of cement pastes', Journal of NonNewtonian Fluid Mechanics, vol. 230, pp. 31-42.

Barnes, H.A., 1997, 'Thixotropy-a review', Journal of Non-Newtonian Fluid Mechanics, vol. 70, pp. 1-33.

Cruz, N., Peng, Y. 2016, 'Rheology measurements for flotation slurries with high clay contents - A critical review', Minerals Engineering, vol. 98, pp. 137.

Dullaert, K., Mewis, J. 2006, 'A structural kinetics model for thixotropy', Journal of Non-Newtonian Fluid Mechanics, vol. 139, pp. 21-30.

Liu, J.Z., Wang, R.K., Gao, F.Y., Zhou, J.H., Cen, K.F. 2012, 'Rheology and thixotropic properties of slurry fuel prepared using municipal wastewater sludge and coal'. Chemical Engineering Science, vol. 76, no. 28, pp. 1-8.

Liu, Q.S., Lu, C.B., Liu, B., Lu, X.L. 2014, 'Research on rheological behavior for cement grout considering temperature and hydration time effects', Chinese Journal of Rock Mechanics and Engineering, vol. 33, pp. 3730-3740.

Møller, P.C.F., Mewis, J., Bonn, D. 2006, 'Yield stress and thixotropy: on the difficulty of measuring yield stresses in practice', Soft Matter, vol. 2, no. 4, pp. 274-283.

Mujumdar, A., Beris, A.N., Metzner, A.B. 2002, 'Transient phenomena in thixotropic systems', Journal of Non-Newtonian Fluid Mechanics, vol. 102, no. 2, pp. 157-178.

Qian, Y., Kawashima, S. 2016, 'Flow onset of fresh mortars in rheometers: Contribution of paste deflocculation and sand particle migration', Cement and Concrete Research, vol. 90, pp. 97-103.

Roussel, N. 2006, 'A thixotropy model for fresh fluid concretes: Theory, validation and applications', Cement and Concrete Research, vol. 36 , no. 10 , pp. 1797-1806.

Roussel, N., Ovarlez, G., Garrault, S., Brumaud, C. 2012, 'The origins of thixotropy of fresh cement pastes', Cement and Concrete Research, vol. 42, no. 1, pp. 148-157.

Usui, H., 1995, 'A thixotropy model for coal-water mixtures', Journal of Non-Newtonian Fluid Mechanics, vol. 60, pp. $259-275$.

Wu, D., Fall, M., Cai, S.J. 2013, 'Coupling temperature, cement hydration and rheological behaviour of fresh cemented paste backfill', Minerals Engineering, vol. 42, pp. 76-87. 